\title{
CARACTERÍSTICAS DE LOS ENSAYOS CLÍNICOS AUTORIZADOS EN EL PERÚ, 1995-2012
}

\begin{abstract}
Gabriela Minaya $^{1,2, a}$, Duilio Fuentes ${ }^{1,2, b}$, Cristian Obregón ${ }^{1, c}$, Beatriz Ayala-Quintanilla ${ }^{1, d}$, Martín Yagui1 ${ }^{1,3, e}$
RESUMEN

Objetivos. Describir las principales características de los ensayos clínicos (EC) autorizados en el Perú desde 1995 a agosto de 2012. Materiales y métodos. Se realizó un estudio transversal, en el que se revisaron todos los expedientes de protocolos de EC presentados para su evaluación y posible aprobación al INS, cuyos datos forman parte del Registro Peruano de EC. Se realizó el análisis descriptivo de los estados de aprobación de los EC, fase de estudio, especialidad, y otras características afines a ellos. Resultados. Se encontraron 1475 EC, de los cuales $1255(85,1 \%)$ fueron autorizados. De un EC registrado el año 1995 se incrementó a 176 ensayos presentados en el 2008, disminuyendo en el 2011 a 128 ensayos. Entre los EC aprobados, el 64,1\% corresponden a EC en fase III. Oncología (22,4\%), Infectología (15,5\%) y Endocrinología (12,7\%) fueron las especialidades más estudiadas y solo el 1.19\%, corresponde a enfermedades tropicales desatendidas. Los hipoglicemiantes orales, antivirales de uso sistémico y antineoplásico fueron los medicamentos más estudiados. La industria farmacéutica transnacional fue el principal patrocinador $(87,1 \%)$ y ejecutor de ensayos clínicos $(62,3 \%)$ los cuales se realizan mayormente en Lima. Conclusiones. Los ensayos clínicos que se realizan en el país son principalmente en enfermedades no transmisibles y son estudios en fase III. La industria farmacéutica constituye el principal patrocinador. Solo el 1.2\%, corresponde a enfermedades tropicales desatendidas, lo cual muestra la poca atención a los problemas de salud de poblaciones en situaciones de vulnerabilidad.
\end{abstract}

Palabras clave: Ensayos clínicos como asunto; Investigación clínica; Utilización de medicamentos; Organizaciones Proveedor-Patrocinador; Regulación y fiscalización en salud; Perú (fuente: DeCS BIREME).

\section{CHARACTERISTICS OF CLINICAL TRIALS AUTHORIZED IN PERU: 1995-2012}

\section{ABSTRACT}

Objective. To identify main characteristics of clinical trials (CT) authorized by the National Institute of Health (INS) of Peru from 1995 to August 2012. Material and methods. Cross-sectional study, which reviewed all records of CT submitted for review and possible approval by INS, whose data are part of the Peruvian Registry of Clinical Trials. We performed a descriptive analysis of the states of the CT approval and other characteristics. Results. 1475 clinical trials were assessed, of which 1255 (85.1\%) were authorized. From 1 clinical trial officially registered in 1995, its quantity increased to 176 trials submitted in 2008, and then, declined to 128 in 2011. Among the approved CT, 64.1\% was in Phase III. Oncology (22.4\%), infectious diseases (15.5\%) and endocrinology (12.7\%) were the most studied specialties and a only $1.2 \%$ were for neglected tropical diseases. The oral hypoglycemic drugs, systemic antiviral and antineoplastic agents were the most studied investigational products. The transnational pharmaceutical industry was the main sponsor $(87.1 \%)$ and executors $(62.3 \%)$ of clinical trials were mostly in Lima. Conclusions. Clinical trials in Peru mainly focus on noncommunicable diseases and phase III studies. The pharmaceutical industry is the main sponsor. A very small number of authorized clinical trials in Peru were for neglected tropical diseases that shows little attention to the health problems in vulnerable populations.

Key words: Clinical trials as topic; Biomedical research; Clinical research; Drug utilization; Provider-Sponsored Organizations; Health care coordination and monitoring; Peru (source: MeSH NLM).

\section{INTRODUCCIÓN}

Los ensayos clínicos (EC) aleatorizados y los metaanálisis se encuentran en la posición más alta de la jerarquía de la evidencia científica ${ }^{(1)}$. Es importante diferenciar investigación clínica, como un concepto más general de intervención en seres humanos ${ }^{(2)}$,con lo específico que significa los ensayos clínicos con medicamentos ${ }^{(3)}$. En el caso de los ensayos clínicos es imprescindible la participación de los seres humanos ya

\footnotetext{
Dirección General de Investigación y Transferencia Tecnológica, Instituto Nacional de Salud. Lima, Perú.

Red Latinoamericana y del Caribe de Bioética, Organización de las Nacionaes Unidas para la Educación, la Ciencia y la Cultura (UNESCO). Montevideo, Uruguay.

Ministerio de Salud. Lima, Perú.

a Médico infectóloga máster en Bioética; ${ }^{\mathrm{b}}$ médico internista máster en Bioética; ${ }^{\mathrm{c}}$ licenciado en Estadística; ${ }^{\mathrm{d}}$ médico Ginecoobstetra máster en Salud Pública $\mathrm{PhD}$ en Medicina; ${ }^{\mathrm{e}}$ Médico epidemiólogo

Recibido: 24-10-12 Aprobado: 31-10-12

* Los datos preliminares de este estudio, forman parte de la tesis para optar al título de especialista en Medicina Interna realizada por Duilio Fuentes “Ensayos clínico en el Perú (1995-2006)”. Realizada en la Universidad Nacional Mayor de San Marcos; 2008.
}

Citar como: Minaya G, Fuentes D, Obregón C, Ayala-Quintanilla B, Yagui M. Características de los ensayos clínicos autorizados en el Perú, 1995-2012. Rev Peru Med Exp Salud Publica. 2012;29(4):431-6. 
sea en estudios etiológicos, diagnósticos y terapéuticos, dando soporte a la evidencia de un conocimiento nuevo. Tales investigaciones revisten especial importancia por las implicancias bioéticas que conlleva la participación de personas (sujetos en investigación) quienes utilizarán productos en investigación cuyo grado de eficacia y seguridad no ha sido aún completamente determinado. Por lo tanto, el estado debe garantizar en todo momento el respeto de los derechos individuales, dignidad y autonomía de los sujetos en investigación, por los riesgos reales y potenciales, y por los errores prevenibles existentes en todo proceso de investigación.

Por ende, los EC en el Perú deben ser realizados en el marco de las Buenas prácticas clínicas ${ }^{(4)}$ y el Reglamento de ensayos clínicos ${ }^{(5,6)}$ que incorporan estándares éticos y científicos de calidad establecidos para diseñar, conducir, registrar e informar los estudios clínicos que involucran seres humanos. Asimismo, como estado miembro de la Organización de las Naciones Unidas para la Educación Ciencia y Cultura (UNESCO) desde el año 1946, es clara nuestra adherencia a la Declaración Universal sobre Bioética y Derechos Humanos 2005 (7) que reconoce la importancia de la libertad de la investigación científica y las repercusiones beneficiosas del desarrollo científico y tecnológico, siempre y cuando se asegure el respeto pleno a la dignidad humana, la protección de los derechos humanos y las libertades fundamentales.

La regulación de los EC en el Perú era responsabilidad de la Dirección General de la Salud de las Persona hasta diciembre de 2002; luego, es el Instituto Nacional de Salud (INS) quien asume su rol regulador a inicios del año $2003{ }^{\left({ }^{(8)}\right.}$. Así, luego de reuniones participativas con los patrocinadores de EC, comités de ética en investigación, Dirección General de Medicamentos, Insumos y Drogas (DIGEMID), entre otros, se logra la aprobación y publicación del Reglamento de ensayos clínicos en el Perú, que establece que el INS es la autoridad encargada a nivel nacional de velar por el cumplimiento de las normas que rigen la autorización y ejecución de los ensayos clínicos ${ }^{(5,6)}$.

En estos casi diez años de ejercicio regulatorio, la realización de EC en el Perú ha mostrado un ascenso significativo en la cualificación, tanto en sus procesos de evaluación para su autorización como a los procesos de seguimiento y vigilancia. Sin embargo, no existe ninguna información sistematizada previa sobre los estudios clínicos que se realizan en el Perú en cuanto a: ¿qué se investiga?, ¿quién patrocina y ejecuta estos estudios en el país?, y ¿en qué centros de investigación se realizan estos estudios? entre otros aspectos. El presente estudio tiene por objetivo conocer la situación actual de los ensayos clínicos en el Perú durante el periodo 1995 hasta agosto de 2012, identificando el número de ensayos clínicos, fases, enfermedade de investigación, productos en investigación, los patrocinadores, los ejecutores y las instituciones de investigación donde se desarrollan los ensayos clínicos.

\section{MATERIALES Y MÉTODOS}

\section{DISEÑO DEL ESTUDIO}

Este es un estudio transversal que señala las principales características de los ensayos clínicos autorizados en el Perú que fueron presentados por los patrocinadores a diferentes instancias del Ministerio de Salud desde el año 1995 hasta agosto de 2012. Se realizó la revisión de todos los expedientes de ensayos clínicos en dicho periodo de estudio, en dos etapas: 1) Verificación y revisión de los expedientes que se encuentran físicamente en el archivo de la Oficina General de Investigación y Transferencia Tecnológica (OGITT) del Instituto Nacional de Salud transferidos por la Dirección General de Salud de las Personas desde el año 1995 hasta el año 2002, y 2) Revisión de expedientes de ensayos clínicos y del actual Registro Peruano de Ensayos Clínicos (REPEC) desde el año 2003 hasta agosto de 2012, periodo que corresponde al tiempo en que la OGITT es la autoridad reguladora de ensayos clínicos en el Perú.

Como criterios de inclusión se consideró lo siguiente: 1) Ensayo clínico presentado para autorización por los patrocinadores en el periodo de estudio; 2) Ensayos clínicos que se encuentran bajo custodia del Instituto Nacional de Salud en el archivo de la OGITT o registrados en el REPEC.

\section{RECOLECCIÓN DE DATOS}

Con la información recopilada se elaboró una base de datos en Excel con las variables por ensayos clínicos: fase de estudio; especialidad estudiada; centro de investigación donde se ejecutan los ensayos clínicos; enfermedad según la Clasificación Internacional de Enfermedades décima versión, (CIE-10); productos en investigación según el Sistema de Clasificación Anatómica; Terapéutica y Química (ATC); patrocinadores y ejecutores.

Las categorías de evaluación de los EC incluyen: estudios presentados; estudios aprobados; no aprobados; sin efecto, o en estado de evaluación. Los trámites sin efecto incluyen los EC que fueron presentados pero que el patrocinador desistió, abandonó o suspendió la evaluación.

\section{ANÁLISIS DE DATOS}

En el análisis estadístico se estudió la distribución de cada una de las variables para mostrar medidas de tendencias y frecuencias mediante tablas y gráficos. Se describen además, las características de los EC en las especialidades 
médicas más frecuentes. Se complementa la presentación con tablas o figuras para la evaluación de tendencias. El análisis se realizó en Excel 2010.

\section{ASPECTOS ÉTICOS}

Se mantuvo siempre la confidencialidad de la información respecto a la información de los protocolos de investigación, sin revelar directamente el nombre de todas las instituciones involucradas, así como la propiedad intelectual de los respectivos protocolos. Los datos que se describen son regularmente presentados en el portal web del Instituto Nacional de Salud (www.ins. gob.pe) como parte de las funciones de transparencia regulatoria, por ello no se solicitó consentimiento a los investigadores para presentar esta información.

\section{RESULTADOS}

Desde enero del año 1995 hasta agosto de 2012 se presentaron 1475 protocolos de EC para la evaluación de su autorización, de ellos 1255 (85,1\%) fueron autorizados; $78(5,3 \%)$ no lo fueron, y $96(6,5 \%)$ fueron resueltos como trámites sin efecto; mientras que 46 (3,1\%) protocolos de EC aún se encuentran en estado de evaluación; en la Figura 1 se puede apreciar la distribución de estos EC por año, donde se aprecia que hubo una tendencia de crecimiento en la presentación de ensayos clínicos hasta el año 2008 con 176 EC presentados, disminuyendo hasta el 2011, con 128 EC presentados.

Del total de EC autorizados, 779 (62\%) ya han sido ejecutados y actualmente 476 EC se encuentran activos o en ejecución desde el año 2000. El mayor número de autorizaciones corresponde a EC en Fase III, con $64,1 \%$ (802) de los 1251 EC; seguida de estudios en Fase II con un 21,1\% (265); solo 2,5\% (31) de los EC corresponden a estudios de Fase I.

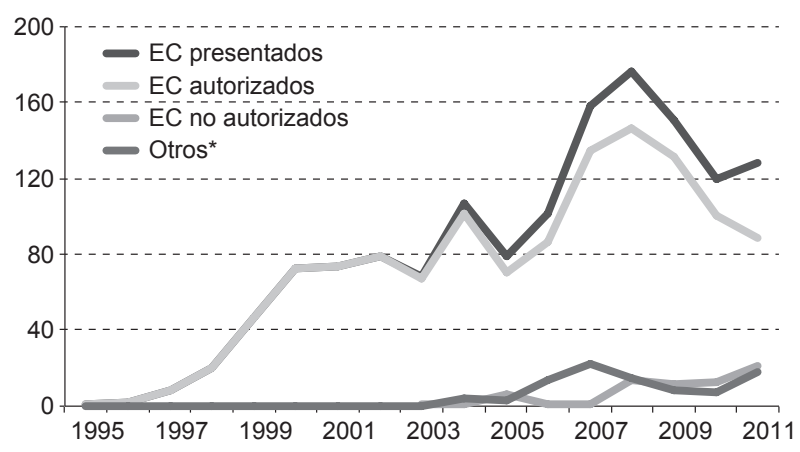

Figura 1. Distribución de ensayos clínicos (EC) según tipo de autorización durante el periodo 1995- 2011.

* Otros: incluye trámites dejados sin efecto por el patrocinador o en estado de evaluación.
De los 1255EC autorizados se identificó 22 especialidades médicas, el $70 \%$ se desarrolla en cinco especialidades: Oncología (22,4\%); Infectología (15,5\%); Endocrinología $(12,7 \%)$; Cardiología $(9,1 \%)$ y Neumología $(8,7 \%)$ (Figura $2)$. Información complementaria sobre los productos de investigación evaluados está disponible en la sección de Anexos (www.ins.gob.pe/rpmesp).

La especialidad con mayor número de EC es Oncología, se encontró 281 EC distribuidos en más de 60 enfermedades CIE-10, entre las que destacan la neoplasia maligna de mama, leucemia mieloide crónica y neoplasias malignas de bronquios y pulmón. Los tipos de antineoplásicos que más se ha estudiado según la clasificación ATC fueron los inhibidores de la tirosina quinasa $(15,9 \%)$, seguido por los anticuerpos monoclonales $(7,8 \%)$, antimetabolitos $(1,8 \%)$ y por la terapia endocrina con inhibidores enzimáticos $(1,8 \%)$.

En Infectología se encontró 194 EC, evaluando más de 70 enfermedades $\mathrm{CIEI}-10$, en donde se destaca la enfermedad por VIH como la más estudiada, seguida de otras como candidiasis, infección bacteriana de tipo no especificado, necesidad de inmunización contra enfermedades virales, neumonía bacteriana, tuberculosis respiratoria, infección local de la piel y del tejido subcutáneo. El producto en investigación más estudiado corresponde a los antivirales de uso sistémico para la enfermedad por VIH (23,9\%), seguido por los antibacterianos (5,6\%), los antimicóticos $(2,6 \%)$ y los antituberculosos $(2,0 \%)$.

En Endocrinología, de 159 EC el $75 \%$ se realizó en diabetes mellitus no insulino dependiente $(71,7 \%)$ y obesidad $(3,1 \%)$. Los hipoglicemiantes orales son los productos de investigación más estudiados según la clasificación ATC, que incluye los receptores activadores de la producción de peroxisomas $(13,8 \%)$, las incretinas $(11,9 \%)$ y los inhibidores de la dipeptidil peptidasa $(11,9 \%)$.

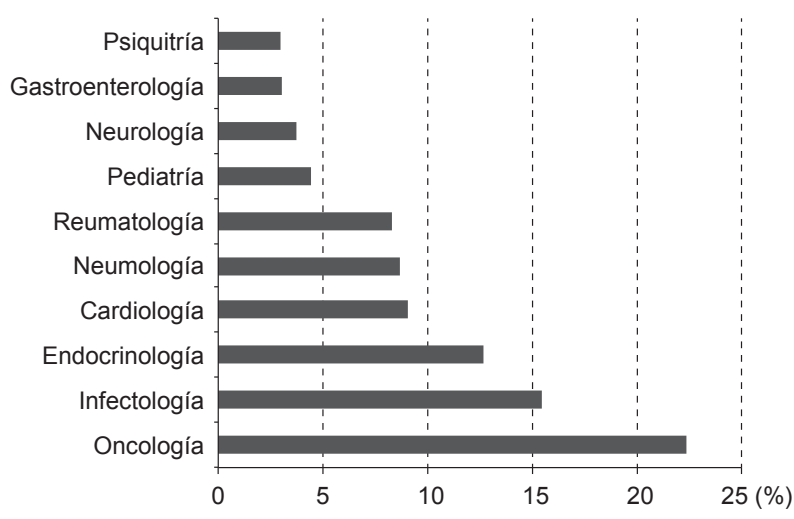

Figura 2. Ensayos clínicos autorizados según especialidad durante el periodo 1995 - 2012. 
En Cardiología se desarrolló 114 EC, la mayoría relacionados con hipertensión esencial $(32,5 \%)$ seguida de fibrilación auricular, enfermedad cardiaca isquémica aguda, hipertensión arterial pulmonar e hiperlipidemia mixta. Los agentes que actúan sobre el sistema renina angiotensina fueron los productos en investigación más estudiados $(11,4 \%)$ de un total de 62 productos.

En Neumología se contabilizó un total de 109 EC, destacando aquellos relacionados con el asma y la enfermedad pulmonar obstructiva crónica. Se encontró que el antagonista del receptor de leucotrienos fue el producto en investigación más estudiado según la clasificación ATC (11,0\%).

Solo el $1,2 \%$ de los EC ( $n=15)$ corresponden a enfermedades tropicales desatendidas (ETD) como leishmaniasis (8), malaria (3), cisticercosis (2), bartonelosis (1) y fiebre amarilla (1). El último ensayo clínico autorizado con estas enfermedades fue en el año 2009.

El 87,2\% (1094) de los EC autorizados fueron patrocinados por la industria farmacéutica transnacional. Así mismo, la industria farmacéutica transnacional fue la encargada de la ejecución de 62,3\% (782) de los EC aprobados, seguido de las CRO (Contract Research Organization) en un $17,6 \%$ (221) y a las instituciones y asociaciones de investigación 9,6\% (121). Solo dos EC $(0,2 \%)$ fueron patrocinados; y cinco $(0,4 \%)$, ejecutados por universidades nacionales (Tabla 1 ).

Las instituciones de investigación donde se ejecutan los ensayos clínicos corresponden en su mayoría a instituciones públicas localizadas en Lima (47\%, principalmente en hospitales de EsSalud), seguido de cerca por las instituciones privadas (46,5\%, principalmente en clínicas). En otras regiones la ejecución de ensayos clínicos es menor (6,5\%).

\section{DISCUSIÓN}

Cada vez más EC son ejecutados a escala mundial, y un tercio se realizan en países en desarrollo; en el Perú desde 1995 hasta más de la mitad del 2012 se han presentado al ente regulador 1475 protocolos EC, con predominio de investigaciones sobre enfermedades no transmisibles, preferentemente en la especialidad de Oncología y los estudios de fase III; asimismo, la industria farmacéutica constituye el principal patrocinador y ejecutor de ensayos clínicos, y existe un crecimiento sostenido del desarrollo de ensayos clínicos en instituciones privadas.

Entre 1910 y 1970 la investigación en enfermedades tropicales endémicas fue fructífera, se desarrollaron los agentes tripanomicidas y antiamebiásicos en los años treinta, la cloroquina en los años cuarenta y cincuenta y los
Tabla 1. Distribución de los ensayos clínicos autorizados según los tipos de patrocinador y ejecutor durante el periodo $1995-2012$.

\begin{tabular}{|c|c|c|}
\hline & N. ${ }^{\circ}$ & $(\%)$ \\
\hline \multicolumn{3}{|l|}{ Patrocinador } \\
\hline Industria farmacéutica transnacional & 1094 & $(87,2)$ \\
\hline Instituciones de investigación y tecnología extranjeras & 76 & $(6,1)$ \\
\hline Grupos cooperativos académicos & 50 & $(4,0)$ \\
\hline Universidades extranjeras & 21 & $(1,7)$ \\
\hline Industria farmacéutica nacional & 4 & $(0,3)$ \\
\hline Asociaciones civiles nacionales & 2 & $(0,2)$ \\
\hline Universidades peruanas & 2 & $(0,2)$ \\
\hline${ }^{*}$ Otros & 6 & $(0,5)$ \\
\hline \multicolumn{3}{|l|}{ Ejecutor } \\
\hline Industria farmacéutica transnacional & 782 & $(62,3)$ \\
\hline $\mathrm{CRO}$ & 221 & $(17,6)$ \\
\hline $\begin{array}{l}\text { Instituciones y asociaciones de investigación } \\
\text { nacionales }\end{array}$ & 144 & $(11,5)$ \\
\hline Persona jurídica & 49 & $(3,9)$ \\
\hline Universidades nacionales & 32 & $(2,5)$ \\
\hline Asociaciones civiles nacionales & 9 & $(0,7)$ \\
\hline Industria farmacéutica nacional & 9 & $(0,7)$ \\
\hline Hospitales nacionales & 7 & $(0,6)$ \\
\hline ** Otros & 2 & $(0,2)$ \\
\hline
\end{tabular}

antihelmínticos en los años sesenta. Sin embargo, a partir de los años ochenta, las estrategias de mercado cambiaron, con un menor desarrollo de novedades terapéuticas $(30,9 \%)$, en especial sobre enfermedades tropicales (1\%) ${ }^{(9)}$. En nuestro trabajo, del total de EC autorizados solo el $1,2 \%$ corresponde a enfermedades tropicales desatendidas (ETD), las cuales según informes de la OMS se concentran, sobre todo, en las comunidades de extrema pobreza, y afectan a más de 1000 millones de personas en el mundo. Bajo esta perspectiva, observamos cómo la investigación va abandonando determinados campos que son prioritarios en el país, como las enfermedades tropicales e infecciosas, esto debido fundamentalmente al bajo poder adquisitivo, para una inversión de alrededor de 160 millones de dólares y que lleva de ocho a doce años en desarrollarse. Este dato también es coherente con lo publicado por la OMS, que refiere que el $90 \%$ de la inversión en investigación mundial está destinado al 10\% de las enfermedades que afectan a la humanidad, con la que la mayoría de las investigaciones que se realizan en los países periféricos no dan cuenta de sus problemas de salud locales, sino de aquellos que aquejan a los habitantes de los países ricos y generalmente el gasto requerido para pagar las innovaciones terapéuticas o sus patentes deja sin recursos a los países en desarrollo para invertir en investigación local ${ }^{(9)}$.

Estados Unidos, Japón y Alemania ocupan los primeros lugares como países en los que se desarrollan ensayos clínicos. Solo en EE. UU. se realizaron 36312 ensayos 
clínicos (31,5\% del total) durante el período 2007 a 2011, según la plataforma internacional de registro de EC de la OMS (WHO ICTRP). En ese periodo en el Perú se ejecutaron un promedio de $600 \mathrm{EC}$, y al igual que en otros países emergentes se aprecia un aumento en los EC registrados; al contrario, existe un descenso para los EE. UU. y Europa Occidental. Entre los países emergentes, China, India y la República de Corea constituyen los países más activos que participan en ensayos clínicos. A pesar de que se observa la transición en la investigación de ensayos clínicos hacia los países emergentes, los países desarrollados siguen contribuyendo con más del $70 \%$ de los EC registrados a nivel mundial ${ }^{(10)}$. En el período del 2001 al 2009, hubo una disminución de la presentación de los ensayos clínicos a las diferentes autoridades reguladoras europeas en los Países Bajos, Alemania, Francia y el Reino Unido. Mientras que en España e Italia tuvo una tendencia creciente, situación similar a la encontrada en nuestro estudio hasta el año $2008{ }^{(11)}$.

En Latinoamérica, la mayor parte de los EC con medicamentos corresponden a estudios internacionales, multicéntricos y multinacionales, y en la última década el número de pacientes que se incorpora a los estudios clínicos se ha incrementado al igual que los estudios de fases más tempranas de desarrollo. Según el Center Watch (DIA 2002), en el año 2000 la población de sujetos en investigación en Latinoamérica era de 150000 y en EE. UU. de 2300 000, estimando que en el año 2010 la población de sujetos en investigación que participarían en EC en Latinoamérica aumentaría en $1000 \%$, mientras que en EE. UU. solo aumentaría en $40 \%$. Esta tendencia también se ve reflejada en el Perú donde el crecimiento ha sido sostenido desde 1995 al 2008, situación que ha ido decreciendo a partir del 2009 por la crisis económica mundial.

Según un estudio conducido por el Instituto Paulista de Referencia en Oftalmología, en Sao Paulo-Brazil (12) tomando como base los datos publicados sobre EC del ClinicalTrials.gov en el año 2009, encontraron que en las áreas terapéuticas de Endocrinología, Hematología y enfermedades infecciosas había una más alta proporción de EC conducidos en países emergentes (Brasil, Argentina, México, entre los latinoamericanos), coincidiendo con enfermedades como Endocrinología e Infectología con las encontradas en nuestro estudio. Por otro lado en otro estudio, en el cual se identificaron EC en 25 países en el periodo de enero de 2007 al 31 de diciembre de 2011 utilizando la plataforma internacional de la OMS registro de EC (OMS ICTRP) se encontró que las enfermedades más estudiadas fueron cáncer, diabetes y enfermedades respiratorias, lo cual también guarda relación con lo encontrado en el presente estudio, en el cual la enfermedad oncológica ocupa el primer lugar como la más estudiada ${ }^{(10)}$. Asimismo, según las referencias revisadas se demuestra que la fase III es en la que más se realiza EC coincidentemente con nuestro trabajo ${ }^{(12)}$.

Observamos una tendencia creciente de investigaciones en enfermedades no transmisibles como la oncológica, endocrina, cardiológica, entre otras. Esto debido a que durante los últimos años la investigación clínica ha experimentado una tendencia creciente hacia la internacionalización. La ola de mundialización de los mercados ha contribuido en gran medida a que los países en vías en desarrollo hayan participado en investigaciones financiadas por las grandes industrias farmacéuticas.

Una observación importante es que en todo este tiempo se han desarrollado pocas moléculas innovadoras y la mayoría son repeticiones de las ya existentes, las llamadas "me-too" drugs. Un importante estudio publicado por Chirac y Torreele (13), encontró que entre las 1556 drogas desarrolladas y registradas por las empresas farmacéuticas entre 1974 y 2004, apenas diez se dirigían a enfermedades exclusivas de los países en desarrollo. Angell M. (14) refiere que del estudio de 415 nuevos medicamentos desarrollados y registrados por la FDA entre 1998 y 2002, apenas el 32\% constituyen nuevas entidades moleculares y $14 \%$ son innovaciones.

Deotro lado, para agilizarel desarrollo de los EC, la industria farmacéutica cambió los centros médicos académicos por organizaciones de mercado para el crecimiento de ganancias, conocidos como las CROs (Organizaciones de Investigación por Contrato, por sus siglas en inglés) y las SMOs (Site Managment Organization). En 1991, el $80 \%$ de la inversión de la industria para EC fue para los centros médicos académicos, cambiando la figura en el año 1998, donde bajó a 40\% ${ }^{(15)}$. En el estudio también se observa esta tendencia en el número de investigaciones ejecutadas por CROs en los últimos años

Las CROs pueden subcontratar a las SMOs para la organización de la red de médicos investigadores, asegurando el rápido enrolamiento de los pacientes. Estas organizaciones proveen del soporte administrativo a los investigadores. En este estudio observamos que las CROs ocupan el segundo lugar en la ejecución de EC en el Perú con un $17,6 \%$. En nuestro estudio algunas Instituciones y asociaciones de investigación nacional, las personas jurídicas y las asociaciones civiles nacionales, se comportan muchas veces como SMOs y han entablado una lucha competitiva con las $C R O$ s para obtener contratos con la industria farmacéutica y en otras ocasionas mediante Grants de investigación para conducir estudios clínicos.

La ejecución en el Perú de los EC por las empresas farmacéuticas transnacionales ha tenido una tendencia decreciente desde el año 2008, en comparación con el 
número de EC ejecutados por CROs desde el año 2003, en el que apareció la primera CRO en el Perú. Asimismo, se delega principalmente la ejecución de los EC a instituciones privadas de investigación nacionales como lo son los institutos de investigación y asociaciones civiles, y a empresas privadas. Pese al evidente crecimiento de los centros de investigación privada, principalmente clínicas y otros centros privados (centros médicos, consultorios e instituciones privadas) tanto en Lima como en las demás regiones (principalmente Arequipa, Trujillo e Iquitos), aún no se ha superado al de instituciones públicas

En nuestro estudio la principal limitación fue identificar muchos EC dentro de la clasificación de la CIE 10 y las categorías delATC, ya que existen muchas combinaciones de enfermedades y medicamentos. En muchos casos el equipo investigador consensuó colocarlo en una sola categoría según la revisión de los objetivos del ensayo y la identificación por parte de los patrocinadores.

En conclusión, los EC que se realizan en el Perú son mayormente para estudiar enfermedades no transmisibles en elárea de Oncología y evalúan la seguridad y eficacia de productos en investigación. Menos del $2 \%$ corresponden a enfermedades tropicales desatendidas (ETD) como bartonelosis, cisticercosis, leishmaniasis, malaria y fiebre amarilla. Asimismo, durante el periodo evaluado se observa que se ha sufrido una migración considerable de la realización de los EC de las instituciones públicas a las privadas, tendencia que hace necesaria que el control del estado se maximice para garantizar en todo momento el respeto de los derechos individuales, dignidad y autonomía de los sujetos en investigación, por los riesgos reales y potenciales existentes. Por ende, los EC en el Perú, deben ser realizados en el marco de Buenas prácticas clínicas y el Reglamento de ensayos clínicos que incorporan estándares éticos y científicos de calidad, establecidos para diseñar, conducir, registrar y reportar los estudios clínicos que involucran seres humanos.

Contribuciones de autoría: GM y DF han participado en la concepción, diseño y redacción del artículo. BA y MY participaron en la revisión y redacción del artículo. CO realizó la recolección, análisis de datos y asesoría estadística. Todos los autores aprueban la versión final a publicar.

Fuentes de financiamiento: autofinanciado.

Conflictos de Interés: los autores de este estudio son miembros del equipo técnico y directivo de la OGITT y son responsables de la conducción del Registro Nacional de Ensayos Clínicos en el Instituto Nacional de Salud de Perú.

\section{REFERENCIAS BIBLIOGRÁFICAS}

1 Peralta V. Ensayos clínicos, industria farmacéutica y práctica clínica. An Sist Sanit Navar. 2005;28(1):7-16.

2 World Health Organization. Handbook for good clinical research practice. Geneva: WHO; 2002.p. 3

3 Administración Nacional de Medicamentos, Alimentos y Tecnología Médica (ANMAT). Buenas Prácticas de Investigación en Estudios de Farmacología Clínica. Disposición ANMAT/5330/1997. Buenos. Buenos Aires: ANMAT; 1997.

4 International Conference of Harmonization. Guideline for Good Clinical Practice [Internet]. Geneva: ICH; 1996 [citado el 26 de septiembre de 2012]. Disponible en: http://www.ich.org/fileadmin/Public Web_Site/ICH_Products/Guidelines/ Efficacy/E6_R1/Step4/E6_R1_Guideline.pdf

5 Perú, Ministerio de Salud. Decreto Supremo 017-2006-SA. Aprueban el Reglamento de Ensayos Clínicos en el Perú. 26 de julio de 2006.

6 Perú, Ministerio de Salud. Decreto Supremo 006-2007-SA. Modifican el Reglamento de Ensayos Clínicos en el Perú. 8 de junio de 2007.
7 UNESCO. Declaración Universal sobre Bioética y Derechos Humanos. Paris: UNESCO; 2005.

8 Perú, Ministerio de Salud. Resolución Ministerial 089-2003-SA/DM. Disponen que procedimiento administrativo establecido en el TUPA del ministerio sea de competencia del Instituto Nacional de Salud. 24 de enero del 2003.

9 Vidal S. ¿Ética o mercado?, una decisión urgente. Lineamientos para el diseño de normas éticas en investigación biomédica en América Latina. En: Genoveva Keyeux (coord.).Ética de la investigación en seres humanos y políticas de salud pública. Número 2. Bogotá: Unibiblos; 2006. p. 191-232.

10 George M, Selvarajan S, Suresh-Kumar S, Dkhar SA, Chandrasekaran A. Globalization of Clinical Trials - Where are we Heading? Curr Clin Pharmacol. 2012. [Epub ahead of print]

11 Hartmann M. Impact assessment of the European Clinical Trials Directive: a longitudinal, prospective, observational study analyzing patterns and trends in clinical drug trial applications submitted since 2001 to regulatory agencies in six EU countries. Trials. 2012;13:53.
12 Alvarenga LS, Martins EN. Biopharmaceutical industry-sponsored global clinical trials in emerging countries. Rev Assoc Med Bras. 2010;56(4):428-33.

13 Chirac P, Torreele E. Global framework on essential health R\&D. Lancet. 2006;13;367(9522):1560-1.

14 Angell M. The truth about the drug companies. How they deceive us and what to do about it. New York: Random House Trade Paperback; 2004.

15 Bodenheimer T. Uneasy alliance--clinical investigators and the pharmaceutical industry. N Engl J Med. 2000;342(20):1539-44.

16 Shuchman M. Comercializing Commercializing clinical trials--risks and benefits of the CRO boom. N Engl J Med. 2007;357(14):1365-9.

Correspondencia: Duilio Fuentes Delgado

Dirección: Calle Cápac Yupanqui 1400, Lima

11, Perú.

Teléfono: (511) 99232749

Correo electrónico:dfuentes@ins.gob.pe 